\title{
Cocaine vs Ecstasy/MDMA: Comparative Effects on Mood and Cognition in Recreational Users
}

\author{
A.C. Parrott ${ }^{*}$, L.J. Evans, J. Howells and R. Robart \\ Department of Psychology, Swansea University, Wales, UK
}

Keywords: MDMA, ecstasy, cocaine, recreation drug - mood, memory, cognition.

\section{INTRODUCTION}

Cocaine powder has overtaken Ecstasy/MDMA in popularity as a recreational party drug in the UK [1]. However there is comparatively little empirical data on its mood or cognitive performance effects. This report describes the findings from three studies comparing Ecstasy users, cocaine powder users, and non-user controls. The three studies involved a variety of mood scales, self-rating questionnaires, and cognitive performance tasks.

\section{METHODS}

In study 1 , the abstinent volunteers comprised 7 Ecstasy/MDMA users, 8 cocaine/ecstasy users, and 9 nonuser controls. The test battery included working memory: consonant updating (updating), trail making (shifting), and random letter generation (inhibition) [2], also supraspan word recall [3], Tromso Social Intelligence Scale [4], and the Dysexecutive Questionnaire [4]. Study 2 involved 10 Ecstasy/MDMA users, 10 cocaine users, and 10 non-user

Table 1. Summary of Cognitive and Mood Findings from Three Independent Studies

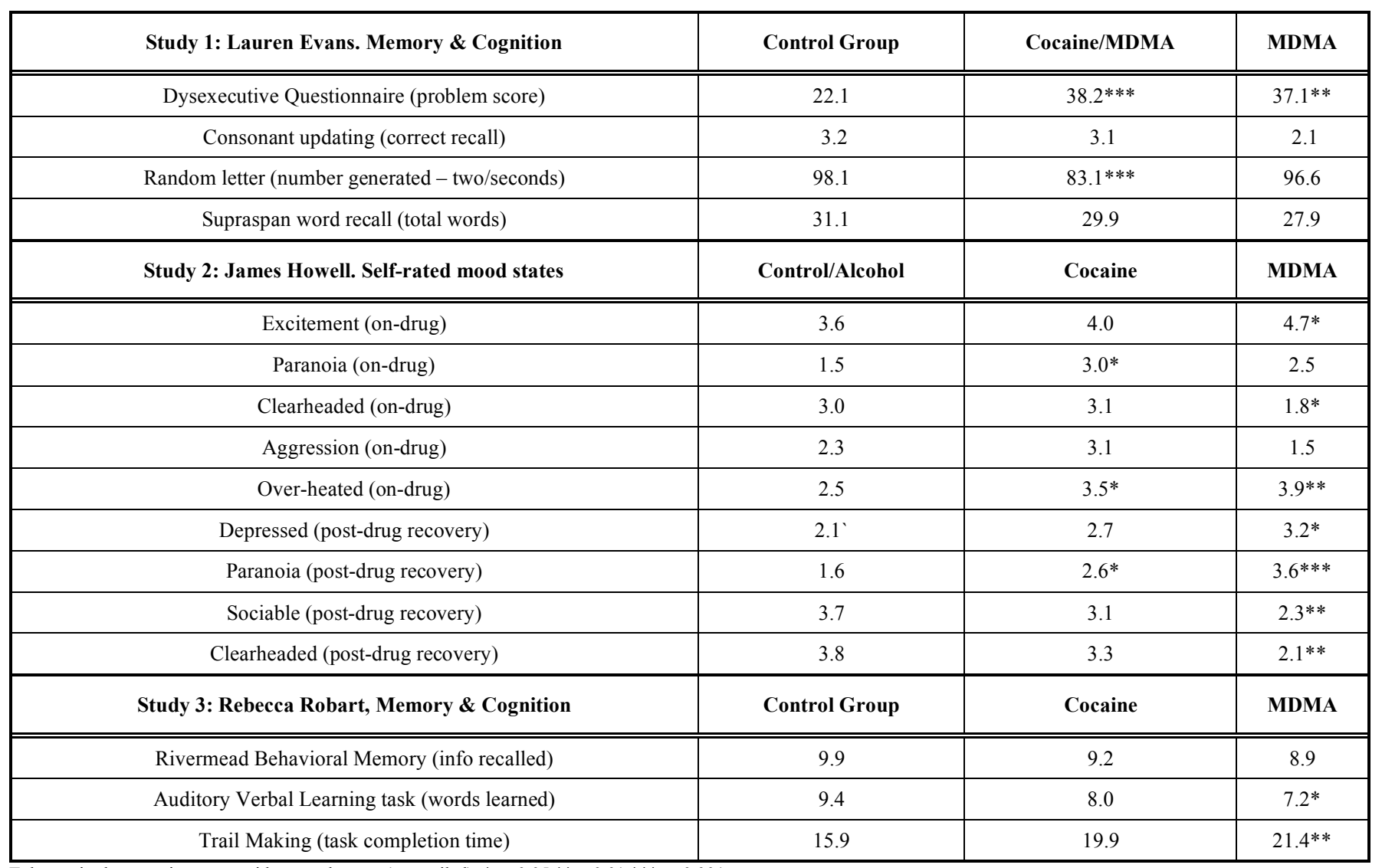

Tukey paired comparison tests with control group (two-tailed): * $\mathrm{p}<0.05^{* *} \mathrm{p}<0.011^{* * *} \mathrm{p}<0.001$.

*Address correspondence to this author at the Department of Psychology, University of Swansea, Swansea SA2 8PP, Wales, UK; Tel: $+44(0) 1792$ 295271; Fax: +44(0)1792 295679; E-mail: a.c.parrott@swansea.ac.uk controls (alcohol drinkers). They were assessed 48 hours after weekend drug use, on a self-rating questionnaire for feelings on-drug, and during post-drug recovery $[5,6]$. Study 
3 involved different groups of 10 Ecstasy/MDMA users, 10 cocaine users, and 10 non-users controls. Participants were assessed 2 and 4 days after weekend drug use, on the Rivermead Behavioural Memory task (paragraph recall), Auditory Verbal Learning (AVLT), and trail making. Model ages across all three studies were 18-30 years.

\section{RESULTS}

In Study 1 Ecstasy users and cocaine/ecstasy users had significantly raised scores on the Dysexecutive Questionnaire. On working memory, cocaine/ecstasy users were more impaired on cognitive inhibition, whereas ecstasy users were slightly more impaired on updating. In Study 2 Ecstasy users were more excited, less clearheaded, and overheated on drug, while cocaine users were more paranoid and overheated. Post-MDMA was associated with depression, paranoia, and reduced sociability. Post-cocaine was associated with paranoia, although to a significantly lesser extent than under MDMA $(p<0.05)$. In Study 3, trail making and AVLT were significantly impaired in Ecstasy users on recovery day 4, while cocaine showed nonsignificant impairments (Table 1). The recovery day 2 group means, and drug condition significance levels, were broadly similar (data not tabulated here).

\section{DISCUSSION}

Cognitive performance was reduced in both Ecstasy/MDMA and cocaine users. On some measures the two groups were impaired to a similar extent (dysexecutive functioning). On one task the cocaine/ecstasy group was more impaired (letter generation). On other tasks the Ecstasy users were more impaired (word recall, verbal learning, trail making). All these comparisons were limited by small sample sizes, and larger studies are required [2, 4, 8]. With the mood data, greater excitement and less clearheadness under MDMA confirm previous findings [7]. Cocaine generated paranoia as expected [8]. Adverse recovery phenomena were pronounced after-MDMA, as in previous studies $[5,6]$. One interesting finding was the significantly higher paranoia post-MDMA than post-cocaine. Another was the comparative data on self-rated thermal stress under both hyperthermic drugs $[9,10]$.

\section{CONCLUSIONS}

We believe this is the first report to empirically compare the mood and cognitive effects of cocaine powder and MDMA. It shows that recreational Ecstasy/MDMA is at least as problematic as recreational cocaine, and may cause worse recovery effects afterwards. Larger studies are however needed to confirm and extend these novel findings.

\section{REFERENCES}

[1] Treadwell SD, Robinson TG. Cocaine use \& stroke. Postgrad Med J 2007; 83: 389-94.

[2] Murphy PN, Wareing M, Fisk JE, Montgomery C. Executive working memory deficits in abstinent ecstasy/MDMA users: a critical review. Neuropsychobiology 2005; 60: 159-75.

[3] Parrott AC. The effects of transdermal scopolamine and four doses of oral scopolamine $(0.15,0.3,0.6,1.2 \mathrm{mg})$ on psychol performance. Psychopharmacology 1986; 89: 347-54.

[4] Reay JL, Hamilton C, Kennedy DO, Scholey AB. MDMA polydrug users show process-specific central executive impairments, and social and emotional judgment processes. J Psychopharmacol 2006; 20: 385-8.

[5] Parrott AC, Lasky J. Ecstasy (MDMA) effects upon mood and cognition; before, during, and after a Saturday night dance. Psychopharmacology 1998; 139: 261-8.

[6] Parrott AC, Lock J, Connor AC, Kissling C, Thome J. Dance clubbing on MDMA and during Ecstasy/MDMA abstinence: prospective neuroendocrine and psychobiological changes. Neuropsychobiology 2008; 57: 165-80.

[7] Davison D, Parrott AC. Ecstasy in recreational users: self-reported psychological and physiological effects. Hum Psychopharmacology 1997; 12: 91-7.

[8] Woicik PA, Moeller SJ, Alia-Klein N, et al. The neuropsychology of cocaine addiction. Neuropsychopharmacology 2009; 34: 111222.

[9] Freedman FR, Johanson C, Tancer ME. Thermoregulatory effects of 3, 4-methylenedioxymethamphetamine (MDMA) in humans. Psychopharmacology 2005; 183: 248-56.

[10] Grandall CG, Vongpatanasin W, Victor RG. Mechanism of cocaine-induced hyperthermia in humans. Ann Intern Med 2002; 136: 785-91.

This is an open access article licensed under the terms of the Creative Commons Attribution Non-Commercial License (http://creativecommons.org/licenses/ by-nc/3.0/) which permits unrestricted, non-commercial use, distribution and reproduction in any medium, provided the work is properly cited. 